\title{
Emittance fluctuation of mesoscopic conductors in the presence of disorders
}

\author{
Wei Ren*, Fuming Xu, and Jian Wang ${ }^{\dagger}$ \\ Department of Physics and the Center of Theoretical and Computational Physics, \\ The University of Hong Kong, Hong Kong, China
}

\begin{abstract}
We report the investigation of the dynamic conductance fluctuation of disordered mesoscopic conductors including 1D, 2D and quantum dot systems. Our numerical results show that in the quasi-ballistic regime the average emittance is negative indicating the expected inductive-like behavior. However, in the diffusive and localized regime, the average emittance is still negative. This disagrees qualitatively with the result obtained from the random matrix theory. Our analysis suggests that this counter-intuitive result is due to the appearance of non-diffusive elements in the system, the necklace states (or the precursor of necklace states in the diffusive regime) whose existence has been confirmed experimentally in an optical system.

PACS numbers: $71.23 .-\mathrm{k} 72.15 . \mathrm{Rn} 74.40 .+\mathrm{k}$
\end{abstract}

The universal behavior of sample-to-sample fluctuation of mesoscopic conductors has attracted intensive theoretical and experimental studies in last two decades [1]. For $\mathrm{dc}$ conductance it is well known that the quantum interference gives rise to a reproducible fluctuation in the coherent mesoscopic structures. When the sample size $L$ is smaller than the coherence length but greater than the elastic mean free path, the system is in the diffusive regime and the conductance fluctuation exhibits a universal behavior with a universal conductance fluctuation (UCF) that is independent of Fermi energy, disorder strength, and system size. The numerical results of conductance fluctuation for various systems 2] are in good agreement with the UCF values obtained from the diagrammatic perturbation theory [1] and the random matrix theory [3]. For ac transport, due to the long range Coulomb interaction, the displacement current should be included in the ac current in a self-consistent manner[4]. Hence it is important to examine the role played by the displacement current in the dynamic conductance fluctuation. For chaotic quantum dots, the fluctuating admittance and capacitance have been studied using random matrix theory [5, 6] . Careful experiments have been carried out to measure this dynamic conductance in clean samples [7, 8]. At low frequencies, the dynamic response of a system to the external bias is characterized by emittance which is the imaginary part of the low frequency admittance and is proportional to the partial density of state (DOS) of the system[4]. Physically, for a conductor with large transmission coefficient, the system responds like an inductor and the emittance is negative while for a conductor with low transmission coefficient the emittance is positive and the system behaves like a capacitor. For a ballistic conductor, the emittance $E_{\mu}$ is found to be $E_{\mu}=-(1 / 4) e^{2} d N / d E$ while for a metallic diffusive wire the average emittance is $E_{\mu}=(1 / 6) e^{2} d N / d E$ where $d N / d E$ is the total DOS of the scattering region [9]. For a chaotic quantum dot, the emittance is also positive from the calculation of random matrix theory [5] showing capacitive-like response. For a disordered 2D waveguide,
De Jesus et al have calculated the emittance distribution using a continuous model [10]. The emittance distribution is Gaussian at weak disorder and crossover to a non-Gaussian distribution with a tail in the negative emittance region at strong disorder.

For low dimensional systems, it has been predicted by Pendry [1] that there exists quasi-extended states known as necklace states in strongly disordered regime. These necklace states are due to the multi-resonance and are very rare events. Recently, the existence of the analogous optical necklace states in disordered $1 \mathrm{D}$ system has been confirmed experimentally in the Anderson localized regime [12]. Since these necklace states come from multiresonance, the electron dwells long time with large DOS in the scattering region giving rise to a large emittance. Thus we expect that the necklace states must have major influence on the average emittance for a disordered 1D system. Motivated by this observation, in this paper we report an investigation of the disorder effects on the emittance and its distribution using tight-binding models for a carbon nanotube system (a quasi-one dimensional system), a two-dimensional waveguide, and a quantum dot. Our numerical results show that different from the previous physical expectation and theoretical results, the average emittance $E_{\mu}$ is always negative even in the localized regime. We attribute this counter-intuitive result as due to the non-diffusive elements in the system, the existence of necklace states (or the precursor of necklace states in the diffusive regime).

Single wall carbon nantoube (SWNT) [13] is a promising candidate for the ac nanoelectronic transistor. In this work, we consider a disordered $(6,6)$ SWNT of 480 atoms connected to two semi-infinite perfect $(6,6) \mathrm{SWNT}$ leads. We employ the conventional nearest-neighbor tight-binding model whose Hamiltonian for the disordered SWNT reads $H=\sum_{i} \epsilon_{i} c_{i}^{\dagger} c_{i}+\sum_{i, j}\left(t_{i, j} c_{i}^{\dagger} c_{j}+h . c.\right)$, where $c_{i}^{\dagger}\left(c_{i}\right)$ is the creation (annihilation) operator for an electron on the carbon site $i, \epsilon_{i}=0$ and $t_{i, j}=3 \mathrm{eV}$ represent the on-site energy and the nearest-neighbor hopping integral, respectively. Static Anderson-type disorder is 


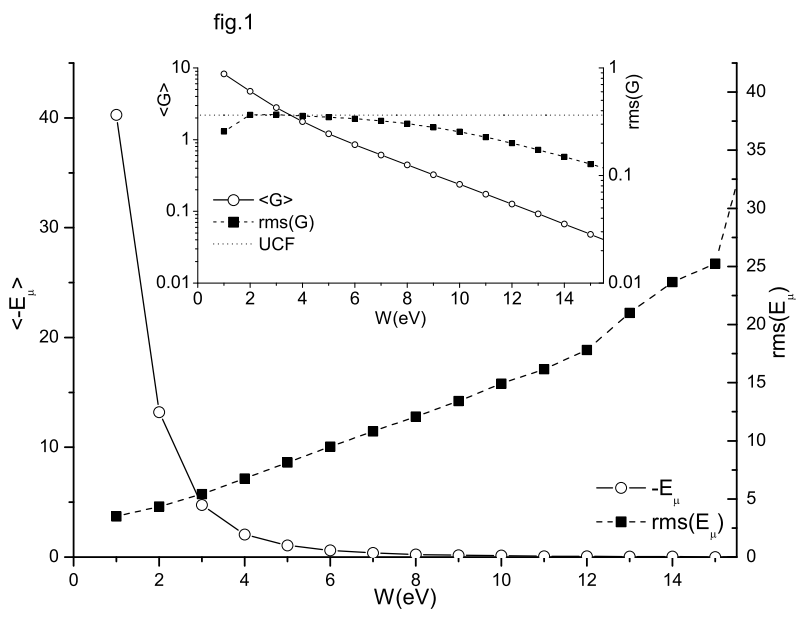

FIG. 1: The mean and rms values of emittance $-E_{\mu}$ at the subband center $\mathrm{E}=3.2 \mathrm{eV}$ for different disorder strengths (one million configurations averaged). Inset shows the corresponding mean and rms values of conductance in unit $2 e^{2} / h$.

added to $\epsilon_{i}$ with a uniform distribution in the interval $[-W / 2, W / 2]$ where $W$ denotes the disorder strength. In this study, we have neglected the strong electron-electron interaction in SWNT.

The conductance of the nanotube is calculated via the Landauer-Buttiker formula, $G(0)=\left(2 e^{2} / h\right) T$ where $T=\operatorname{Tr}\left(\Gamma_{1} \mathrm{G}^{\mathrm{r}} \Gamma_{2} \mathrm{G}^{\mathrm{a}}\right)$ is the transmission coefficicent, $G^{r}=$ $\left[E-H-\Sigma_{1}^{r}-\Sigma_{2}^{r}\right]^{-1}$ is the retarded Green's function of the central disordered SWNT and the relationship between self-energy and linewidth function is $\Gamma_{1,2}=i\left[\Sigma_{1,2}^{r}-\Sigma_{1,2}^{a}\right]$. With the help of density of states given by Green's functions, we can express emittance as 14]

$$
E_{\mu}=-\operatorname{Tr}\left(\operatorname{Im}\left[\mathrm{G}^{\mathrm{a}} \Gamma_{2} \mathrm{G}^{\mathrm{r}} \Gamma_{1} \mathrm{G}^{\mathrm{a}}\right]_{\mathrm{xx}}-\frac{\mathrm{D}_{1 \mathrm{xx}} \mathrm{D}_{2 \mathrm{xx}}}{\mathrm{D}_{1 \mathrm{xx}}+\mathrm{D}_{2 \mathrm{xx}}}\right)
$$

where $D_{\alpha}=G^{r} \Gamma_{\alpha} G^{a}, D_{\alpha x x}=\left[D_{\alpha}\right]_{x x}$, and $[\cdots]_{\mathrm{xx}}$ denotes the diagonal element of the relevant square matrix. The quantities in the first and second terms in Eq.(1) have clear physical meaning. The first term is the partial DOS $d N_{12} / d E$ (up to a sign) describing the DOS of the electron coming from the second lead and exiting the first lead while the second term consists of local DOS $[9]$ and is due to the displacement current. The dynamic response given by $E_{\mu}$ is either positive for small transmission coefficient or negative for large transmission coefficient giving rise to an inductive-like or capacitive-like behavior. For instance, for small transmission coefficent $d N_{12} / d E$ is very small and the sign of the emittance is dominated by the second term which is positive.

We first examine the emittance fluctuation defined by the root mean square (rms) values as $\operatorname{rms}\left(E_{\mu}\right)=$ $\left[\left\langle E_{\mu}^{2}\right\rangle-\left\langle E_{\mu}\right\rangle^{2}\right]^{1 / 2}$, where $\langle\cdots\rangle$ denotes averaging over an ensemble of samples, with different configurations of the same disorder strength. Fig.1 shows the averaged emittance and its fluctuation vs disorder strength with the energy fixed at the subband center. We also plot the average conductance (with unit $2 e^{2} / h$ ) and its fluctuation for comparison. As the disorder increases the system crosses over from quasi-ballistic to diffusive regime. Eventually for strong disorders the nanotube becomes an insulator in the localized regime. The localization length $\xi$ defined as $G=G_{N} \exp (-2 L / \xi)$ is shown in Fig.4a where $G_{N}$ is the conductance in the absence of disorder and $L$ is the system size. We see that the conductance fluctuation in the diffusive regime $W=[2,4]$ (where $\xi>L$ ) approaches to the one dimensional value of $\mathrm{UCF} 0.73 e^{2} / h$ (see the dotted line in the inset of Fig.1) obtained from the diagrammatic perturbation theory [1] and the random matrix theory [3].

The main panel of Fig.1 shows that the average emittance is always negative. This is a very counter-intuitive result since we expect that a conductor with small average conductance gives a capacitive-like behavior corresponding to a positive emittance. It is true that for any specific configuration, the conductance and emittance are well correlated from our numerical result, i.e., large conductance corresponds to negative emittance and small conductance corresponds to positive emittance. The reason that the average emittance gives an inductive-like response is due to the so-called necklace states (or precursor of necklace states in the diffusive regime) that are rare events in the disordered systems. Through these states, electrons can tunnel through the disordered system of size $L$ via multiple scattering. The larger the system size, the smaller the number of necklace states is. The existence of these extended states in localized regime was predicted by Pendry 11]. Different from the single resonant tunneling, the necklace state is a necklace of $n$ quasi-extended states stretching from one site of the disordered sample to other. They occupy only a fraction of sites with fractal dimension $1 / 2$ in $1 \mathrm{D}$ reminiscent of percolation path[11]. The analogous optical necklace states have been observed experimentally in disordered multilayer systems [12] where the high transmission peaks show up in the transmission spectrum while the average of logarithmic of transmission coefficient decays linearly with the thickness. Since the necklace states (multi-resonant states) are rare events, they have very long life time giving rise to a large negative emittance. To make this point clear, we plot in Fig.2 the distribution functions $P\left(E_{\mu}\right)$ versus emittance $E_{\mu}$ at different disorder strengths $W$. For this figure we have used data from 200,000 different random configurations except in panel (h). We see that at low disorder strengths when $W \leq 2$ the distribution is approximately Gaussian. In this disorder range, as the disorder strength increases the values of emittance are all negative and the mean emittance gradually increases towards positive value. For larger disorder strength $W=4$ in panel (d), the distribution 


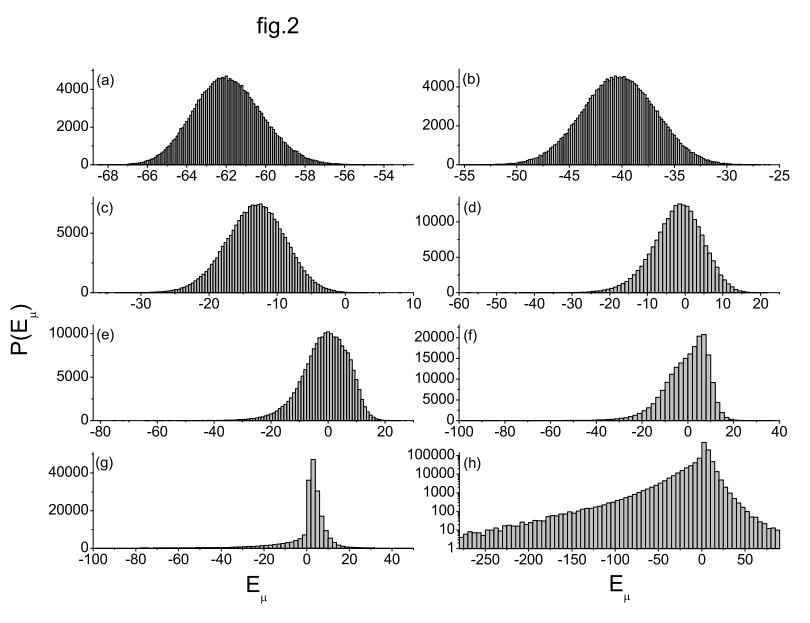

FIG. 2: The histograms of emittance at the subband center $\mathrm{E}=3.2 \mathrm{eV}$ for different disorder strength $\mathrm{W}=0.5,1.0,2.0,4.0$, 5.0, 6.0 and $10.0 \mathrm{eV}$, respectively. Panel (h) is the same as panel $(\mathrm{g})$ but in a log scale of $P\left(E_{\mu}\right)$ with one million configurations.

is still approximately Gaussian but the emittance can be positive while the mean emittance is negative. When $W=5,6$ the distribution deviates gradually from the Gaussian distribution and the mean emittance is around zero but negative. At even stronger disorder $W=10$, the distribution is sharply peaked at positive emittance with a long tail in the negative emittance (see Fig.2h). To be more precise, the distribution is asymmetric with large probability at small positive emittance while the probability of negative emittance is small but remains finite even for large negative emittance resulting a negative averaged emittance.

Why the effect of necklace states has not been seen in the conductance fluctuation but important for the dynamic response? This is because at large disorders, there exists small number of necklace states with small probability. For these necklace states the magnitude of emittance can be very large while the largest possible value of conductance is around one since it is unlikely for all multichannels to tunnel through. Therefore the contribution of necklace states to the average conductance is very small but is very large for the average emittance. For instance, in Fig.2h with $W=10$, the average emittance is -0.11 and total conductance from eleven channels is $G=0.24$. Out of one million configurations, the total probability of these necklace states with emittance $E_{\mu}<-100$ is 0.3 percent. Their contribution to the average emittance and average conductance are, respectively, $E_{\mu}=-0.39$ and $G=0.002$. Clearly, these necklace states are important for average emittance and negligible for average conductance. Without these necklace states the average emittance would be positive. For exactly the same reason, the emittance fluctuation is greatly enhanced due to the existence of these necklace states. We point out that these necklace states exist as long as the system size $L$ is finite. In addition to the quasi-1D system, we have also studied the averaged emittance of a disordered 1D tight-binding chain. Our result indicates that our conclusion remains, i.e., the average emittance is always negative for all disordered strengths.

Note that the necklace states exist in the localized regime and our analysis above on these states is also done in the same regime. Now we wish to argue that the average emittance $E_{\mu}$ is negative in the diffusive regime as well. Since $E_{\mu}$ is a monotonic function of disorder strength, hence $E_{\mu}$ in the localized regime is larger than $E_{\mu}$ in the diffusive regime. The fact that $E_{\mu}$ is negative in the localized regime means that $E_{\mu}$ is negative in all regimes including diffusive regime. Physically, in the diffusive regime, the system is roughly described by a diffusive conductor in parallel with multi-resonant channels connecting the leads (the precursor of necklace states). It is the appearance of these non-diffusive elements in the diffusive system, the precursor of necklace states that changes the sign of $E_{\mu}$ in the diffusive regime from positive to negative.

Now we study the emittance using a tight-binding model for a quantum dot and a $2 \mathrm{D}$ system with disorders. The system size is $L=40 a$ where $a$ is the lattice spacing and two electrodes of width $L_{1}$ are attached to the scattering region. For the quantum dot $L_{1}=L / 4$ while for the $2 \mathrm{D}$ system $L_{1}=L$. The average conductance and average emittance and their fluctuations for the quantum dot and 2D system are depicted in Fig.3 where we have collected one million configurations for each data point. From Fig. 3 we see that conductance fluctuation in the diffusive regime exhibits plateaus with universal values that are close to the theoretical predictions $U C F=0.70 e^{2} / h$ for quantum $\operatorname{dot}[3]$ and $U C F=0.86 e^{2} / h$ for $2 \mathrm{D}$ system [1]. For emittance, our results show that for both the quantum dot and the $2 \mathrm{D}$ system, the emittances are negative independent of disorder strengths that disagrees with the theoretical predictions. The emittance fluctuations for quantum dot (QD) and 2D system are consistent with 1D case.

Our results for the average emittance clearly disagree with the theoretical results obtained from the random matrix theory. This is because in order to use analytic approach, one has to make the following approximation on the second term in Eq.(11) so that all the quantities involved are global,

$$
\operatorname{Tr}\left[\frac{\mathrm{D}_{1 \mathrm{xx}} \mathrm{D}_{2 \mathrm{xx}}}{\mathrm{D}_{1 \mathrm{xx}}+\mathrm{D}_{2 \mathrm{xx}}}\right] \approx \frac{\operatorname{Tr}\left(\mathrm{D}_{1}\right) \operatorname{Tr}\left(\mathrm{D}_{2}\right)}{\operatorname{Tr}\left(\mathrm{D}_{1}+\mathrm{D}_{2}\right)}
$$

It is easy to see that this approximation greatly overestimates the second term in Eq.(1) at large disorders. For weak disorders with large transmission coefficient $D_{1 x x}$ and $D_{2 x x}$ are in the same order of magni- 


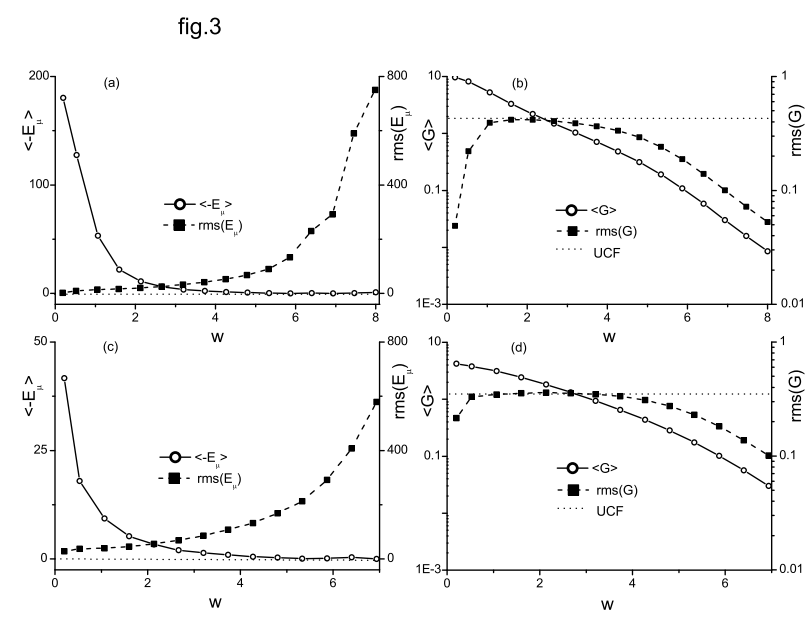

FIG. 3: The mean and rms values of emittance $-E_{\mu}$ of the $2 \mathrm{D}$ system in panel (a) at $E=0.62$ and the QD in panel (c) at $E=3.0$ for different $W$ (one million configurations averaged). The corresponding mean and rms values of conductance in unit $2 e^{2} / h$ are shown in panel (b) and (d), respectively, for the $2 \mathrm{D}$ system and the QD.

tude hence Eq.(2) is a good approximation. However, at strong disorders there is a large mismatch between $D_{1 x x}$ and $D_{2 x x}$ for quite a number of sites $x$. Since $D_{1 x x} D_{2 x x} /\left(D_{1 x x}+D_{2 x x}\right) \approx D_{2 x x}$ when $D_{1 x x}>>D_{2 x x}$ the left hand side of Eq.(2) is approximately given by $\operatorname{Tr}\left[\min \left(\mathrm{D}_{1 \mathrm{xx}}, \mathrm{D}_{2 \mathrm{xx}}\right)\right]$ which is much smaller than the right hand side of Eq.(2) at strong disorders. Using this approximation, our numerical results are shown in Fig.4. We see that, indeed, for both quasi-1D and 2D systems the average emittance with the approximation Eq.(2) (solid square) overestimated the second term in Eq.(1) so that the results are very different from that obtained using Eq.(1). This is because the necklace states have been washed out in Eq.(2). In addition, Fig.4 shows that using approximation Eq.(2) the emittance is positive in the diffusive regime in agreement with the theoretical predictions.

In conclusion, we have studied the fluctuation of emittance and its distribution for disordered 1D, 2D, and quantum dot systems. Our numerical results indicate that the average emittance is always negative independent of disorder strengths. Our findings disagree qualitatively with the theoretical results obtained from the random matrix theory. The disagreement is due to the existence of non-diffusive elements, necklace states or the precursor of necklace states that are important for the dynamic response and negligible for the conductance fluctuation.

This work was financially supported by RGC grant (HKU 7044/04P) from the government SAR of Hong Kong and LuXin Energy Group. Endeavor Australia

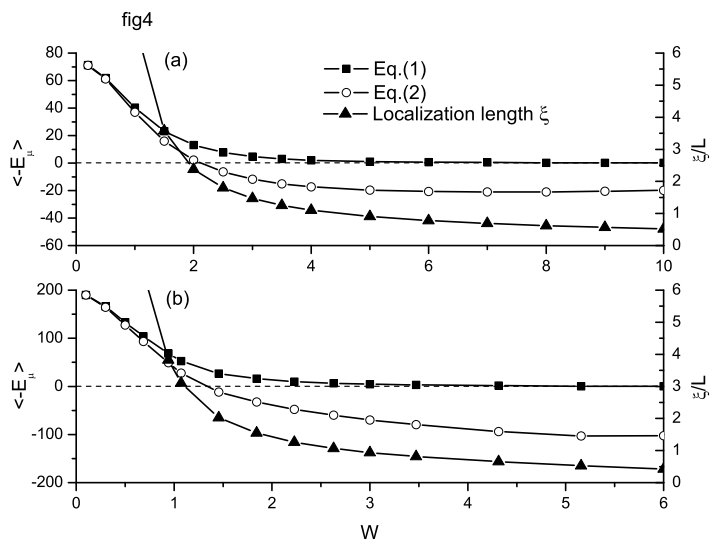

FIG. 4: The average emittance $-E_{\mu}$ and the localization length $\xi / L$ vs $W$ (100 000 configurations averaged) for quasi$1 \mathrm{D}$ and $2 \mathrm{D}$ systems are shown, respectively, in panel (a) and (b). The other parameters are the same as in Fig.1 and Fig.3a.

Cheung Kong Programme sponsored by Cheung Kong Group is also acknowledged (W.R.).

* Present address: Department of Physics, The Hong Kong University of Science and Technology, Clear Water Bay, HK, China

† Electronic address: jianwang@hkusua.hku.hk

[1] B.L. Altshuler, JETP Lett. 41, 648 (1985); P. A. Lee and A. D. Stone, Phys. Rev. Lett. 55, 1622 (1985); P. A. Lee et al, Phys. Rev. B 35, 1039 (1987).

[2] H. Tamura and T. Ando, Phys. Rev. B 44, 1792 (1991); H. U. Baranger and P. A. Mello, Phys. Rev. Lett. 73, 142 (1994); A. Garcia-Martin and J. J. Saenz, Phys. Rev. Lett. 87, 116603 (2001); A. Cresti et al, Phys. Rev. B 69, 233313 (2004).

[3] C.W.J. Beenakker, Rev. Mod. Phys. 69, 731 (1997).

[4] M. Buttiker, J. Phys.: Condens. Matter 5, 9361 (1993).

[5] P.W. Brouwer and M. Buttiker, Europhys. Lett. 37, 441 (1997).

[6] V.A. Gopar et al, Phys. Rev. Lett. 77, 3005 (1996).

[7] J. Regul et al, Physica E 22, 272 (2004).

[8] J. Gabelli et.al., Science 313, 499 (2006).

[9] M. Buttiker and T. Christen, in "Quantum transport in semiconductor submicron structures", p263, edited by B. Kramer, Kluwer Academic Publishers, Netherlands, 1996.

[10] T. De Jesus et al, Phys. Rev. B 62, 10774 (2000).

[11] J.B. Pendry, J. Phys. C 20, 733 (1987).

[12] J. Bertolotti et al, Phys. Rev. Lett. 94, 113903 (2005).

[13] M. Bockrath et al., Science 275, 1922(1997); A. Bachtold et al., Phys. Rev. Lett. 84, 6082(2000); R. Martel et al., Appl. Phys. Lett. 73, 2447(1998).

[14] T. Gramespacher and M. Büttiker, Phys. Rev. B 56, 13026 (1997); W. Ren and J. Wang, Phys. Rev. B 69, 033306 (2004). 